\section{A new solution to dry mouth}

Water can be used as a saliva replacement; however, it does not moisten and lubricate the oral mucosa and teeth adequately - something which is critical to providing relief to patients with dry mouth.

Unlike some other dry mouth products, the entire Oralieve Dry Mouth Relief product range has been formulated with a unique combination of bioactive ingredients and enzymes to provide effective, long-lasting relief for your patients suffering from dry mouth. The soothing formulations help maintain the $\mathrm{pH}$ of the mouth and help supplement the natural protective lubricating systems in saliva.

Oralieve Moisturizing Mouth Gel is a saliva substitute with intensive moisturisers to soothe and lubricate for long lasting relief. Ideal for use at night, Oralieve Moisturising Mouth Gel can be applied as often as required. Oralieve Moisturising Mouth Spray is ideal for use during the day providing on-the-go relief from dry mouth, and freshness.

Oralieve Ultra Mild Toothpaste contains no foaming agents (SLS) and its mild flavours and ingredients are gentle on the mouth. An alcohol-free fluoride mouth rinse is also available with a mild flavour to be gentle for dry mouths.

Patients who are at risk of developing dry mouth include: patients with diabetes, patients on multiple medications, patients with Sjögren's syndrome and patients who have suffered from head and neck cancer. ${ }^{1,2}$ The impacts of dry mouth can be wide ranging and can make chewing, swallowing and talking difficult as well as leading to oral health complications such as caries and gingivitis.

Visit www.oralieve.co.uk to request your sample and for further information on any of the products.

1. National Institute of Dental and Craniofacial Research. Dry mouth (xerostomia). Available at https://www. nidcr.nih.gov/oralhealth/topics/drymouth/ (accessed 20 December 2016).

2. Dirix P, Nuyts S, Van den Bogaert W. Radiation-induced xerostomia in patients with head and neck cancer. Cancer 2006; 107: 2525-2534.

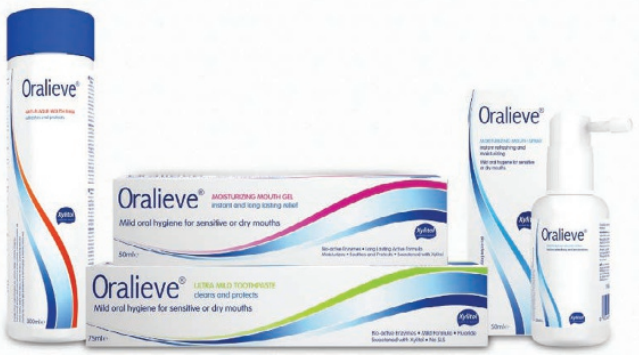

\section{Streamlining UK dental radiology}

Clinical imaging company INFINITT UK have launched a web-based service to streamline digital imaging and reporting workflow in the UK dental radiology community.

INFINITT SmartNet for Dental Radiology is a cloud service optimised to simplify management and exchange of dental images and for radiologists requiring a remote reporting platform. SmartNet enables real-time viewing, sharing and scalable storage of dental images and diagnostic reports. Online worklists provide real-time status updates with integral analytical review tool options.

Associated users in the dental community are invited to log into a subscriber's service via Infinitt's secure portal to submit templated referral requests and supporting history data. It provides an easy route for general dental practitioners to place orders (using a selectable

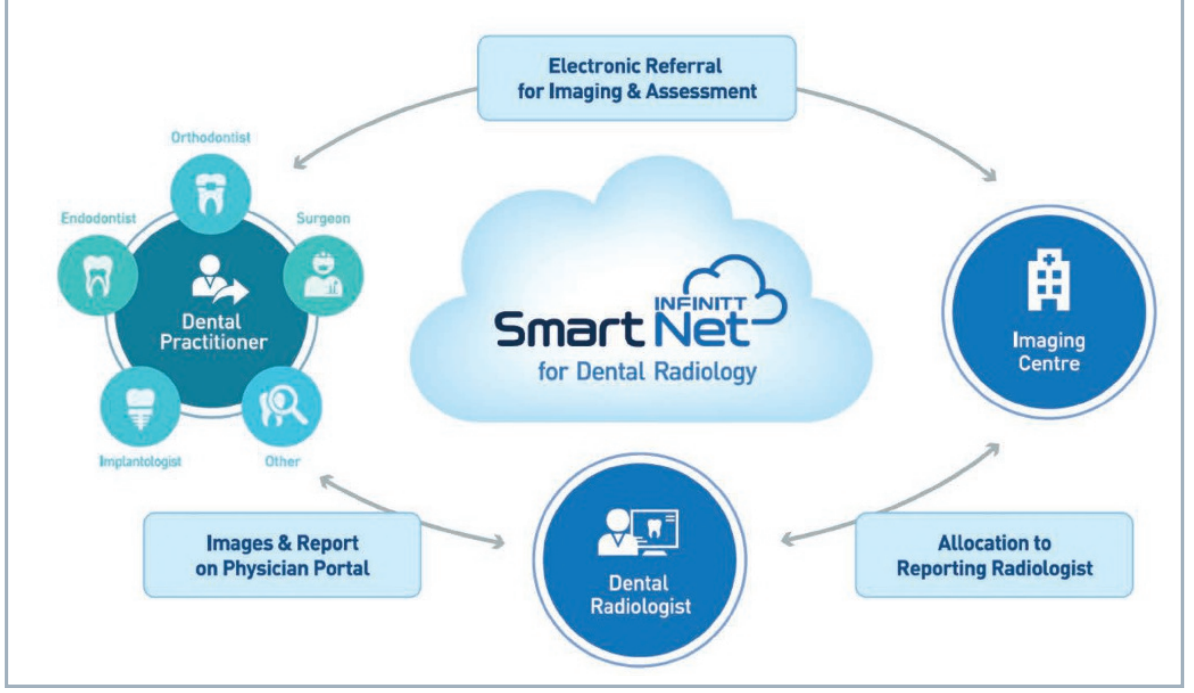

\section{Contemporary solutions for complex challenges}

Nobel Biocare will be among the leading implant product suppliers and manufacturers delivering programmes in the Corporate Forum at the ADI Team Congress 2017.

Don't miss Bertil Friberg's session on 'Treating the compromised patient, what we have learned from 40,000 patients at Brånemark Centre in Gothenburg' or Alessandro Pozzi's 'Using technology to solve complex treatment problem - from design to prosthetics'.

From the UK, Guy McLellan will explore 'Treating complex maxillary situations with digial planning, models and quad zygomas', helping all delegates take their digital workflows to the next level. upload function) and also to review orders and resulting images/reports/comment.

Diagnostic reporting and surgical procedure planning is supported with online viewing and measurement tools plus layout options including FMX templates for all dental image types. It includes Infinitt's own Xelis 3D Dental module for CBCT and Implant Planning.

This hosted platform delivers the same dedicated INFINITT image and information management solution used in NHS dental hospitals and private practice.

INFINITT SmartNet offers a fully automated Referral/Imaging/Report workflow at reduced cost at attractive low cost.

Visit http://www.infinitt.com/cms/ services/cloud-based-service or email sales@ infinitt.co.uk.
Entitled 'Contemporary solutions for complex treatment challenges', the morning's lectures will provide delegates with two hours of verifiable CPD and be delivered by world class professionals in the field.

'Treat more patients better' - don't miss Nobel Biocare's Corporate Forum at the ADI Team Congress 2017, Thursday 2 March from 8-10 am in ICC Capital Suite, Level S Room 2.

For more information about Nobel Biocare, call 0208756 3300, or visit www. nobelbiocare.com. The ADI Team Congress 2017 - 2-4 March - ExCeL London. For more details, please visit www.adi.org.uk/ congress 17 . 